\title{
Numerical approach to a problem of hydroelectric resources management
}

V. A. Bushenkov, M. M. A. Ferreira, A. F. Ribeiro, and G. V. Smirnov

Citation: AIP Conference Proceedings 1558, 630 (2013);

View online: https://doi.org/10.1063/1.4825570

View Table of Contents: http://aip.scitation.org/toc/apc/1558/1

Published by the American Institute of Physics

\section{Articles you may be interested in}

Sufficient conditions of optimality for control of hydro-electric power stations

AIP Conference Proceedings 1558, 586 (2013); 10.1063/1.4825559 


\title{
Numerical Approach to a Problem of Hydroelectric Resources Management
}

\author{
V. A. Bushenkov *, M. M. A. Ferreira ${ }^{\dagger}$, A. F. Ribeiro** and G. V. Smirnov ${ }^{*}$ \\ ${ }^{*}$ Department of Mathematics, University of Évora, Portugal \\ ${ }^{\dagger}$ FEUP, ISR, University of Porto, Portugal \\ ${ }^{* *}$ University of Porto \\ ${ }^{\ddagger}$ Centre of Physics, Department of Mathematics and Applications, \\ University of Minho, Portugal
}

\begin{abstract}
In this paper we consider a simplified model for a system of hydro-electric power stations with reversible turbines. The objective of our work is to obtain the optimal profit of power production satisfying restrictions on the water level in the reservoirs. Two different numerical approaches are applied and compared. These approaches center on global optimization techniques (Chen-Burer algorithm) and on Projection Estimation Refinement method (PER method) used to reduce the dimension of the problem.
\end{abstract}

Keywords: optimal control; global optimization; projection estimation refinement method PACS: $02.30 . \mathrm{Yy} ; 02.40 . \mathrm{Vh} ;$; 87.55.de;88.60.np

\section{INTRODUCTION}

In [5], it is considered a simplified model for a cascade of hydro-electric power stations where some of the stations have reversible turbines. The objective is to optimize the profit of power production. The problem is treated in the framework of optimal control theory and sufficient conditions of local optimality are deduced.

In this paper we consider a cascade with two hydro-electric power stations. The fluxes of water to turbine or pump on each power station are the controls and the maximization of the profit of energy sale is the objective function. The state variables represent the water volumes in reservoirs which are subject to some constraints. The presence of these constraints and the nonconvexity of the cost function contribute to the complexity of the problem.

After the discretization of our problem we obtain a problem of maximization of a indefinite quadratic form subject to linear constraints. The nonconvexity of the cost function enables the existence of several local maxima and the application of global optimization methods is necessary. We use the Chen-Burer algorithm developed to minimize general quadratic form subject to linear constraints in $R^{n}$ (for details, see [4].)

Two different approaches are discussed and compared. In the first one, we directly apply the Chen-Burer algorithm. In the second approach we use a specific structure of the cost function that allows us to reduce the dimension of the problem constructing a projection of the set of feasible solutions onto a subspace of the cost function arguments. Then the Chen-Burer algorithm is applied to the projected low-dimensional problem. After that, an approximate solution to the original discrete problem is obtained solving a simple convex programming problem and is used as an initial guess for local optimization methods.

\section{PROBLEM STATEMENT}

For a cascade with 2 hydro-electric power stations, the dynamics of water volumes, $V_{k}(t), k=1,2$, is described by the following control system $\quad \dot{V}_{1}(t)=A-u_{1}(t), \quad \dot{V}_{2}(t)=u_{1}(t)-u_{2}(t)$,

where the controls $u_{1}(t)$ and $u_{2}(t)$ are the water flows for each reservoir, at time $t$, and $A$ is the incoming flow.

The control variables and the water volumes satisfy the following technical constraints:

$$
V_{k}(0)=V_{k}(T), \quad V_{k}(t) \in\left[V_{k}^{m}, V_{k}^{M}\right], \quad u_{k}(t) \in\left[u_{k}^{m}, u_{k}^{M}\right]
$$


Here $V_{k}^{m}$ and $V_{k}^{M}, k=1,2$, stand for the imposed minimum and maximum water volumes, respectively; $u_{k}^{m}$ and $u_{k}^{M}$, $k=1,2$, are the imposed minimum and maximum the water flows. The objective is to find optimal controls $\hat{u}_{k}(\cdot)$ and respective volumes $\hat{V}_{k}(\cdot)$, that lead to an optimal management of water in the system:

$$
J(u(\cdot), V(\cdot))=\int_{0}^{T} c(t)\left[u_{1}(t)\left(\frac{V_{1}(t)}{S_{1}}+H_{1}-\frac{V_{2}(t)}{S_{2}}-H_{2}\right)+u_{2}(t)\left(\frac{V_{2}(t)}{S_{2}}-H_{2}\right)\right] d t \longrightarrow \max ,
$$

where $c(\cdot)$ is the price of the energy, $H_{k}, k=1,2$, are the liquid surface elevations and $S_{k}, k=1,2$, are the areas of the reservoirs.

In this work we assume that the price $c(\cdot)$ takes constant values $c_{1}$ and $c_{2}$, on the intervals $\left[0, \frac{T}{2}\left[\right.\right.$ and $\left[\frac{T}{2}, T\right]$, respectively. With this $c(\cdot)$ and replacing the control variables by equivalent expressions obtained from the control system, we write (see [5] for details) the cost function as

$$
\begin{gathered}
J(u(\cdot), V(\cdot))=-\frac{A c_{1}}{S_{1}} \int_{0}^{T / 2} V_{1}(t) d t-\frac{A c_{2}}{S_{1}} \int_{T / 2}^{T} V_{1}(t) d t+H_{1}\left(c_{2}-c_{1}\right) V_{1}(0)+\frac{c_{2}-c_{1}}{2 S_{1}} V_{1}^{2}(0)-H_{1}\left(c_{2}-c_{1}\right) V_{1}\left(\frac{T}{2}\right) \\
-\frac{c_{2}-c_{1}}{2 S_{1}} V_{1}^{2}\left(\frac{T}{2}\right)+H_{2}\left(c_{2}-c_{1}\right) V_{2}(0)+\frac{c_{2}-c_{1}}{2 S_{2}} V_{2}^{2}(0)-H_{2}\left(c_{2}-c_{1}\right) V_{2}\left(\frac{T}{2}\right)-\frac{c_{2}-c_{1}}{2 S_{2}} V_{2}^{2}\left(\frac{T}{2}\right) .
\end{gathered}
$$

The discretization of this problem is constructed in the following way. Let $N$ be an even number. We define new variables $x$ and $y$ as

$$
\begin{gathered}
x=\left[V_{1}(0), V_{1}\left(\frac{N}{2}\right), V_{2}(0), V_{2}\left(\frac{N}{2}\right)\right], \text { and } \\
y=\left[V_{1}(1), \cdots, V_{1}\left(\frac{N}{2}-1\right), V_{1}\left(\frac{N}{2}+1\right), \cdots, V_{1}(N-1), V_{2}(1), \cdots, V_{2}\left(\frac{N}{2}-1\right), V_{2}\left(\frac{N}{2}+1\right), \cdots, V_{2}(N-1)\right],
\end{gathered}
$$

The cost function takes the form

$$
I(x, y)=\langle a, x\rangle+\langle b, y\rangle+\langle x, Q x\rangle \rightarrow \min ,
$$

where $a$ and $b$ are appropriate vectors gathering the linear part of the cost relative to $x$ and $y$ and $Q$ is an appropriate matrix defining the quadratic part of cost function.

The constraints of the problem are translated into

$$
\begin{gathered}
V_{i}(k) \in\left[V_{i}^{m}, V_{i}^{M}\right], \text { for } k=0, \cdots, N-1 \text { and } i=1,2, \\
V_{1}(k)+A-V_{1}(k+1) \in\left[u_{1}^{m}, u_{1}^{M}\right], \text { for } k=0, \cdots, N-2, \\
V_{2}(k)+V_{1}(k)+A-V_{1}(k+1)-V_{2}(k+1) \in\left[u_{2}^{m}, u_{2}^{M}\right], \text { for } k=0, \cdots, N-2, \\
V_{1}(N-1)+A-V_{1}(0) \in\left[u_{1}^{m}, u_{1}^{M}\right], \quad V_{2}(N-1)+V_{1}(N-1)+A-V_{1}(0)-V_{2}(0) \in\left[u_{2}^{m}, u_{2}^{M}\right] .
\end{gathered}
$$

\section{NUMERICAL METHODS}

We directly apply the Chen-Burer algorithm to the discretized problem. This algorithm combines a finite branching based on the first order Karush-Kuhn-Tucker conditions with polyhedral-semidefinite relaxations of completely positive programs (see [4]). The time taken by this algorithm is very long and we consider another approach. Before applying Chen-Burer algorithm we reduce the dimension of the problem using the Projection Estimation Refinement method (PER) from [1].

With this method the orthogonal projection $P$ of a polytope $X$ onto a subspace is approximated by a sequence of polytopes $P^{0}, P^{1}, \ldots, P^{k}, \ldots$ that tend to $P$, and $P^{k} \subset P$ for all $k$. The number of vertices of polytopes increase by one at each iteration. Every next polytope is constructed on the basis of the previous one using procedures of computing the support functions for the projection $P$ and Fourier-Motzkin convolution method ([2]). In [3] a robust algorithm for solving this problem was proposed.

For approximating polyhedra, two descriptions are constructed simultaneously, one as a set of their vertices and the other as a system of linear inequalities. Knowing inequalities of internal approximating sets and the values of the corresponding support functions, it is easy to find external approximating sets $\bar{P}^{0}, \bar{P}^{1}, \ldots, \bar{P}^{k}$, which contain the projection $P$, i.e., $P^{k} \subset P \subset \bar{P}^{k}$ for all $k$. 

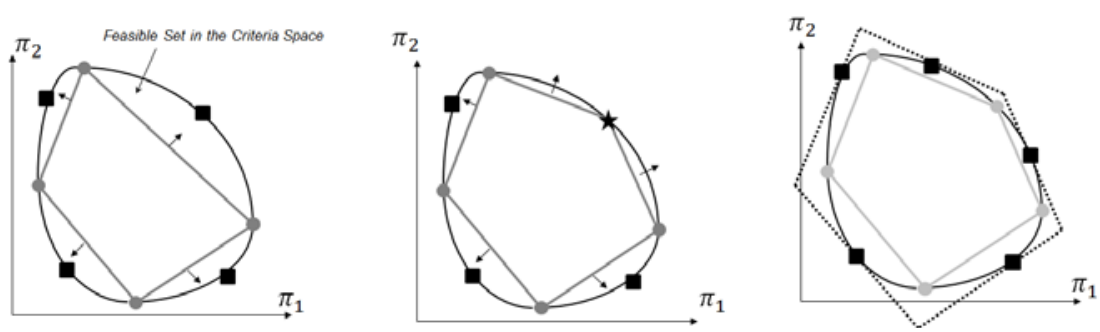

FIGURE 1. (Left) 1 st iteration - the initial set; (Center) 2nd iteration - the most distant new point $\star$ is included into the convex hull; (Right) Internal estimation (convex hull of vertices) and external estimation (described by constructed support-planes);

The first two pictures in Fig.1 show an iteration on the constructing process of orthogonal projection. Computational details and a discussion of these techniques for polyhedral approximation can be found in [6]. Returning to our discretized problem, define a new variable $z=\langle b, y\rangle$ and exclude the variable $V_{1}(1)$ which can be written as

$$
V_{1}(1)=-\left(\frac{s_{1}}{A c_{1}} z+V_{1}(2)+\ldots+V_{1}\left(\frac{N}{2}-1\right)+\frac{c_{2}}{c_{1}}\left(V_{1}\left(\frac{N}{2}+1\right)+\ldots+V_{1}(N-1)\right)\right) .
$$

The cost function (1) can be expressed in terms of $x, z$

$$
\langle\bar{a}, \bar{x}\rangle+\langle\bar{x}, \bar{Q} \bar{x}\rangle \rightarrow \min ,
$$

where $\bar{x}=(x, z), \bar{a}=(a, 1)$, and $\bar{Q}=\left(\begin{array}{cc}Q & 0 \\ 0 & 0\end{array}\right)$.

The projection of the set of feasible solutions onto the subspace of variables $\left(V_{1}(0), V_{1}(N / 2), V_{2}(0), V_{2}(N / 2), z\right)$ is constructed using PER method. With this projection and cost function (2) we get an optimization problem in $R^{5}$. The Chen-Burer algorithm is applied to this problem and a solution is obtained. A simple convex programming problem is then used to get an approximate solution to original discrete problem. Finally, this approximate solution is used now as an initial guess when applying a local optimization method.

Next we present numerical results obtained when the following data for parameters of problem are considered

$$
\begin{gathered}
V_{1}^{m}=86.7, V_{1}^{M}=147, V_{2}^{m}=48.3, V_{2}^{M}=66, u_{2}^{m}=0, u_{2}^{M}=0.8316, u_{1}^{m}=-0.3456, u_{1}^{M}=0.4392, \\
N=T=24, c_{1}=2, c_{2}=20, H_{1}=3, H_{2}=1, A=0.1589, s_{1}=81.7, s_{2}=44.5 .
\end{gathered}
$$

\section{Results obtained with direct use of Chen-Burer Algorithm}

Directly application of Chen-Burer algorithm to the discretized problem gives a solution shown in Fig. 2.
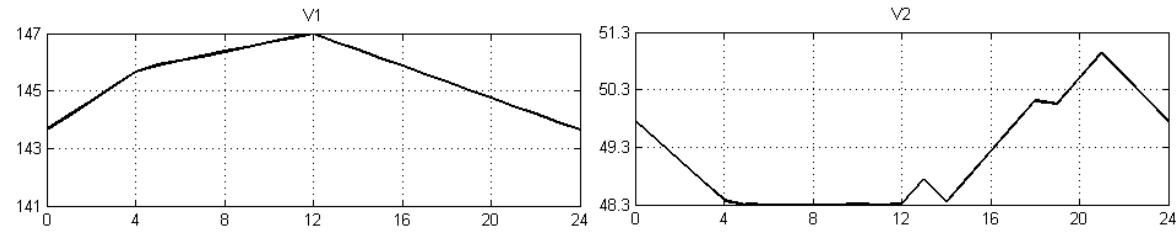

FIGURE 2. Results for the Discretized problem

This global solution has cost $308.918 €$. The execution time is 24 hours.

\section{Results with PER method}

Using PER method [1] we get a feasible set for the projected problem (exterior approximation with 15 inequalities). The Chen-Burer algorithm is applied to this problem and the obtained solution is

$$
\hat{\bar{x}}=(\hat{x}, \hat{z})=[140.66,147,48.30,49.16,-68.18 .]
$$


An approximate solution to the discretized problem is obtained solving the following convex quadratic programming problem: $\quad$ minimize $\quad\|\Pi(y)-\hat{x}\|^{2}, \quad$ s.t. $\quad A y \leq b, \quad A_{e q} y=b_{e q}, \quad L B \leq y \leq U B$,

where $y=\left(V_{1}(0), V_{1}(1), \cdots, V_{1}(N-1), V_{2}(0), V_{2}(1), \cdots, V_{2}(N-1)\right), \quad \Pi(y)=\left(V_{1}(0), V_{1}(N / 2), V_{2}(0), V_{2}(N / 2)\right)$, and $\hat{x}=\left(\hat{V}_{1}(0), \hat{V}_{1}(N / 2), \hat{V}_{2}(0), \hat{V}_{2}(N / 2)\right)$. We used the function QuadProg from the Matlab.
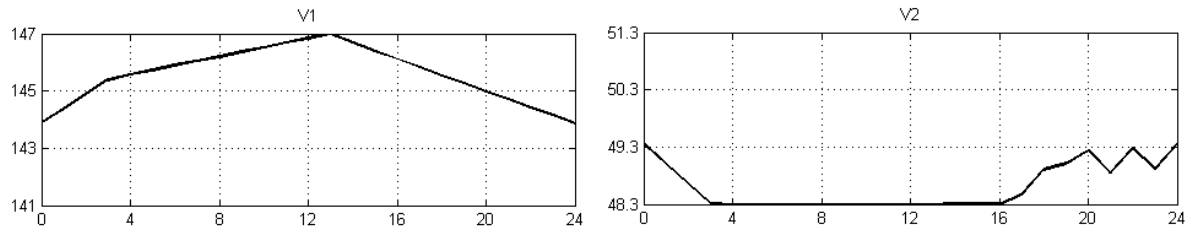

FIGURE 3. Approximate solution

This solution together with $\hat{x}$ is then used as an initial guess for the optimization package from [7]. The final result is presented in Fig. 4
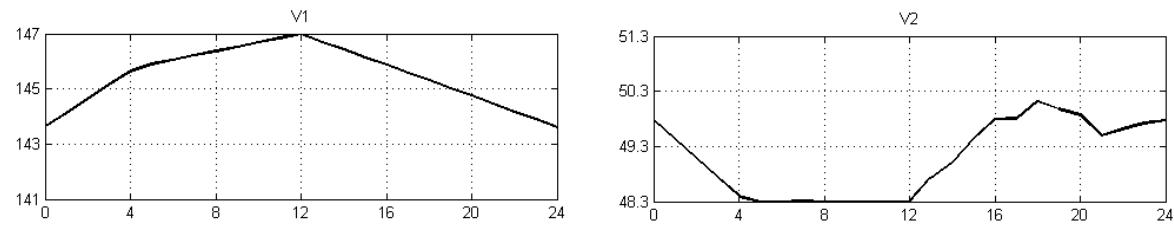

FIGURE 4. Final results with new approach

The cost associated to this solution is the same. The comparison of two approaches is presented in table 1 .

TABLE 1. Comparison of methods

\begin{tabular}{lcc}
\hline & 1st approach & 2nd approach \\
\hline & $\bullet$ Chen - Burer Algorithm \\
(directly) & $\begin{array}{l}\bullet \text { PER } \bullet \text { Chen - Burer Algorithm } \\
\bullet \text { QuadProg } \bullet \text { Local optimization }\end{array}$ \\
\hline Total time execution & 24 hours & $1.48 \mathrm{~min}$ \\
\hline
\end{tabular}

Note that different optimal trajectories on the 2 approaches are due to non-uniqueness of solution to this problem.

\section{ACKNOWLEDGMENTS}

This work has been partially supported by the EU 7th Framework Prog. [FP7-PEOPLE-2010-ITN] grant agr.64735SADCO, FCT project PTDC/EEA-CRO/116014/2009 and project FCOMP-01-0124-FEDER-028894, Ref.FCT PTDC/EEI-AUT/1450/2012

\section{REFERENCES}

1. V.A. Bushenkov, An iteration method of constructing orthogonal projections of convex polyhedral sets, U.S.S.R. Comput. Maths. Math. Phys., 20(3), pp.1-5 (1995)

2. S.N. Tchernikov, Lineare Ungleichungen, Deutcher Verlag der Wissenschaften, Berlin (1971)

3. O.L.Chernykh, Construction of the convex hull of a finite set of points when the computations are approximate, U.S.S.R. Comput. Maths. Math.Phys., 28(5), pp. $71-77$ (1988)

4. J. Chen and S. Burer, Globally solving nonconvex quadratic programming problems via completely positive programming, Math. Prog. Comp., 4(1), pp.33-52 (2012)

5. M.M.A. Ferreira, A.F. Ribeiro and G.V. Smirnov, Sufficient Conditions of Optimality for a Cascade of Hydro-Electric Power Stations, long abstract submited to ICNAAM'2013(2013)

6. A.V. Lotov, V. A. Bushenkov, and G. K. Kamenev, Interactive Decision Maps: Approximation and Visualization of Pareto Frontier, Springer (2004)

7. G. Smirnov and V. Bushenkov, Curso de Optimização: Programação Matemática,Cálculo das Variações, Controlo Óptimo, Escolar Editora (in Portuguese)(2005) 